\title{
Achieving Inclusion with Contextualized User-Sensitive Design ${ }^{1}$
}

\author{
LI Fang $^{1}$ and DONG Hua ${ }^{2}$ \\ ${ }^{1}$ Tongji University, 1239 Siping Road, Shanghai 200092, P.R. China \\ franklifangetongji.edu.cn \\ ${ }^{2}$ Loughborough University, Epinal Way, Loughborough, Leicestershire LE11 3TU, UK \\ h.dong@lboro.ac.uk
}

\begin{abstract}
Users and contexts in human-computer interaction systems often have great diversity, which limits the inclusion provided by a single design. Existing user-sensitive design and contextual design methodologies have made useful attempts to pay attention to diversities, but they still cannot provide sufficient design basis for adapting to dynamically changing user capabilities, needs and usage contexts. Based on the analysis of the interaction between the behavior model and various elements in the human-computer system, this paper constructs a contextualized user-sensitive design framework, and studies the diversity and changing factors in the human-computer system from the two basic dimensions of the user and the context. In order to reflect the multi-dimensional dynamic characteristics of users, the authors propose a new user research and analysis tool, the generalized user balance sheet, for user-sensitive design, and takes a questionnaire case to reveal the huge differences of users' needs in different contexts. Based on the contextualized usersensitive design framework, this paper shows the basic methods and essential system elements of user-sensitive design and contextualized design, as well as the possibility of combining the two for inclusive design.
\end{abstract}

Keywords: Inclusive Design, User Sensitive Design, Contextual Design, User Centered Design.

\section{Introduction}

When using languages to express certain concepts, we are often constrained and influenced by the language itself. For example, when we express the concept of 'same', we usually only recognize that the referred objects are the same when they are nearly $100 \%$ identical. However, when we need to judge 'different', the standard seems to be much lower. $10 \%$ or even less inconsistency is enough to make us believe that there are significant differences. As a result, in a complex system of the world, the same / different equilibrium point that we think may be distorted greatly. When we think that most individuals in a group are different, in fact there are only small and partial diversities between different individuals. This difference in semantic concepts requires us to be extremely cautious when choosing words to describe concepts. Therefore, this paper chooses 'diversity' and 'similarity' to represent the difference of users from two different perspectives in the same dimension.

Diversities and similarities are like head and tail of a coin, and their complementarity forms a unity of opposites. In most user groups with a certain scale, it is almost impossible to have absolute similarities and diversities if they are based on individual users. Therefore, diversities and similarities must exist at the same time. The combination of the two can fully reflect the overall needs and characteristics of users. The similarity of users enables designers to build core features around which products or services can be scaled up. For this reason, it is usually the focus of user research. This is of course understandable, but if we only focus on the common characteristics of users and ignore the understanding and research of diversities, then the resulting design proposal may easily lead to

1 The final authenticated version is available on line at https://doi.org/10.1007/978-3-030-23560-4_9 
either a lack of inclusiveness or failure to provide inclusive results in a reasonable, effective and sustainable manner.

\section{Design for Diversities}

The characteristics of things are the sum of diversities and similarities. Since we have made clear the complementarity of diversities and similarities, we may as well regard 'commonness' as a special form of diversities, which is convenient for us to start from diversities and solve the problem of how to maximize design inclusiveness under the resource constraints.

\subsection{Behavior Model and Human-Machine System}

For design research, user behavior is one of the research focuses that cannot be avoided. Users interact with products through certain activities to achieve a certain purpose or meet a certain demand, which is always completed in a certain context. Gonzalez and Morer summarized the relationship among the user, product, contexts and activity into the system structure as shown in Fig. 1 from the system view of ergonomics[1]. Fogg's behavior model proposes that behavior is the product of motivation, ability and trigger factors. The behavior can only occur if the actor has sufficient motivation and execution ability and is triggered to execute the behavior[2]. Economists attribute human behavior to making choices among many alternative behaviors in order to eliminate discomfort[3]. Although the expressions and theoretical backgrounds of theories are different, the laws and principles expounded by them are basically the same. Eliminating discomfort is actually the fundamental goal and motivation of human beings, specific behaviors and trigger factors are created by the context of making choices, and making choices among many optional behaviors is based on the evaluation and comparison of the execution ability of different behaviors. For an activity, the higher the actor's ability, the simpler it is to complete the activity and the lower the cost. At the same time, the greater the benefits and value of the behavior, the stronger the motivation. Therefore, if the ability dimension in Fogg's behavior model is transformed into cost through reciprocal, and the motivation dimension corresponds to the interest demand, we can easily reduce Fogg's behavior model to the basic assumption of economics for individuals to pursue self-interest maximization and typical demand curve.

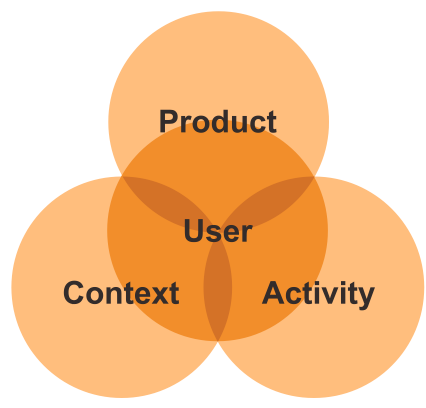

Fig. 1. UCAP map[1].

In the UCAP map of Fig. 1, although the elements constituting the design of human-machine system revolve around users, there is no clear mutual relationship, which reflects that ergonomic research only classifies and roughly locates these elements, and lacks in-depth discussion on the role and logical structure of these elements in the design process. This paper regards products, users, situations and activities as four independent vertices, and connects them with lines with directional arrows to form a tetrahedral structure (Fig. 2). If this tetrahedron is rotated in a three-dimensional 
space, we can examine its interaction with different elements from the perspective of a certain vertex. At the same time, the diagram also indicates the relationship between this structure and design, external environment and goal, and then we will analyze one by one.

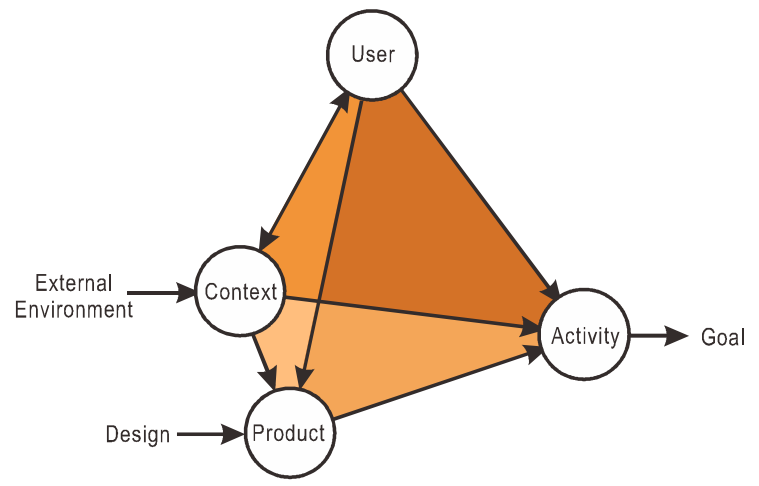

Fig. 2. Human-machine system tetrahedron.

Product. A product is a direct design object. It can be any artifact, service or artificial environment. Designers provide users with choices and means for targeted activities by shaping products, and thus influence and help users achieve goals through activities. As the substantial carrier of supply, the product can be independent or a component in a larger system. Of course, product design will inevitably be constrained by the supply capacity, but this is an inherent constraint outside the human-machine system. Under this constraint, the basis for helping designers to make design decisions in the human-machine system mainly comes from users and contexts. Once the design of a product is determined, it will often have a certain stage or a certain degree of stability. However, due to the diversity and variability of users and contextual factors, if the product cannot provide enough choices and tools for users to complete the target activities, it means that the problem of inclusiveness will arise.

On the other hand, the relationship between specific products and context-user-activity plane is also unstable. Although designers assume that the product is a part of the human-machine system when designing products, in fact, the design of a single product often faces many alternative choices. Only by maintaining the continuous competitive advantage against alternative solutions can the product keep its position as a member of the human-machine system, which is especially difficult with the change of users and contexts.

Activity. Although we often emphasize taking users as the center in design research and practice, users' needs and goals are always achieved by using products to conduct certain activities in specific contexts. Activities are initiated by users, influenced and restricted by contexts, and are mainly completed through the use of products. Users, products and specific contexts are all indispensable elements of activities, and these three elements have their own motivations.

From the perspective of products, activities can be regarded as an indirect design object. Designers can further support and influence users' activities by product design. From the user's point of view, the main actor of the activity is the user him/herself, that is to say, the user is the final decisionmaker and executor of the activity. From the perspective of the context, the context is the inducement that the external environment works on the user and leads to the activity. The external environment here includes all stakeholders of the system in this context.

Since activities are directly related to goals, ideal activities are aimed at the common goals of users with their own needs, providers represented by products and stakeholders in specific context. However, product providers must have their own interests and try to influence users' behavior through products based on these interests. Users also hope to maximize their own interests by using 
products in games with stakeholders under specific contexts, which makes the relationship between product providers and users very subtle. As a bridge between product providers and users, whether designers want to mediate the conflicts of interest between the two or have obvious tendentiousness often depends on the identity and position of designers, and activities are the outcome of such competing relationships. Designers need to study and understand the users and contexts, imagine the activities that users need to carry out according to the demand goal, and then respond through product design.

User. Users are the group who will choose appropriate product functions to engage in certain activities according to their own situation and the context at that time. The reason why it is called group is that although products and services may be designed for personal use, in most cases, a large number of individual users need to share the same design, so the diversity formed by user diversities puts forward requirements and challenges for the inclusiveness of product design.

Because users and designers have the same sense of autonomy, in human-machine system, the user is actually the core leading factor in system operation. Under certain limited conditions, when the context forces the user to decide to complete the highly challenging activity goal, even if the product fails to provide sufficient and strong support, the user will still try his best to overcome the difficulty of the activity and strive to achieve the goal by creatively using the product or investing more user resources. Similarly, when the system has limited constraints on users, if the products are not usable, users are completely likely to give up solutions based on existing products and adopt more competitive systems to meet their own needs and goals.

Context. Some researchers believe that contexts can also be designed, while we tend to regard contexts as an objective existence independent of the product (design objects) and user. If we can accurately understand that the context is the result of interaction between the user and the external environment, it is not difficult to find that the context itself is not controlled by design. From the perspective of designers, contexts can be found, observed, predicted and evaluated, thus providing a basis for product design.

Contextual factors often change from time to time, from place to place, from person to person, and we can classify these changes into two categories. One is the so-called force majeure, that is, the contextual factors that the user can only passively accept. The contextual factors act on the user unilaterally, forcing the user to respond. Force majeure mentioned here, of course, is also relative. It means that the influence of users on the external environmental factor is very small and negligible. For example, when passengers are waiting for the train at the platform, the arrival time of the train is force majeure for the users, who have to accept it passively. Another kind of context change is the result of interaction between users and the external environment, i.e. both users and the external environment have considerable influence on the context. Changes in either side may cause context changes, such as driving on urban roads, and the context difference at this time will be shown as a function of changes in the external environment and users themselves.

The lack of support for users to engage in certain activities and contexts in product design certainly weakens users' ability to meet their needs. However, due to the existence of user factors, users' needs are not necessarily rigid and irreplaceable. Other activities in other contexts are likely to make up for dissatisfaction with demand and insufficient design support in some contexts. Therefore, it is necessary to look at the role and function of contexts from a systematic perspective in addition to microscopic immersion experience. 


\subsection{Design Framework and Principles}

The research into design has increasingly shifted from industrial design for traditional hardware products to interaction design for smart devices, software and Internet services. A fundamental change in this trend is that the design object itself has gained the possibility and potential to meet and adapt to differentiated needs. Unlike printed labels, mechanical structures, physical switches, clearly defined functional specifications and interactive logic on traditional devices, in an emerging interaction design interface, the typeface, font size, spacing and layout of the page can be adjusted at any time according to user needs; the brightness and color of the screen can be automatically adjusted according to environmental conditions with ambient light sensors; and customized content and commodity information can be pushed to different registered users based on algorithms and big data, or even the iteration of the product can be performed through over-the-air (OTA) upgrade. It means that the diversity of users and contexts has become a design opportunity rather than a design challenge.

Design tools, methods and theories, such as the persona[4] and user profile[5] for analyzing user behavior patterns and preference characteristics, responsive design[6] and adaptive design[7] for different screen sizes and resolutions, as well as contextual design for user-centered system[8], have been created around how to make the full use of this potential to design a more inclusive experience for interaction. These tools, methods and theories propose solutions to a certain kind of factors that cause demand diversities and changes, but they are still not enough to systematically and comprehensively solve the problems caused by complex contexts and user diversity in the design practice.

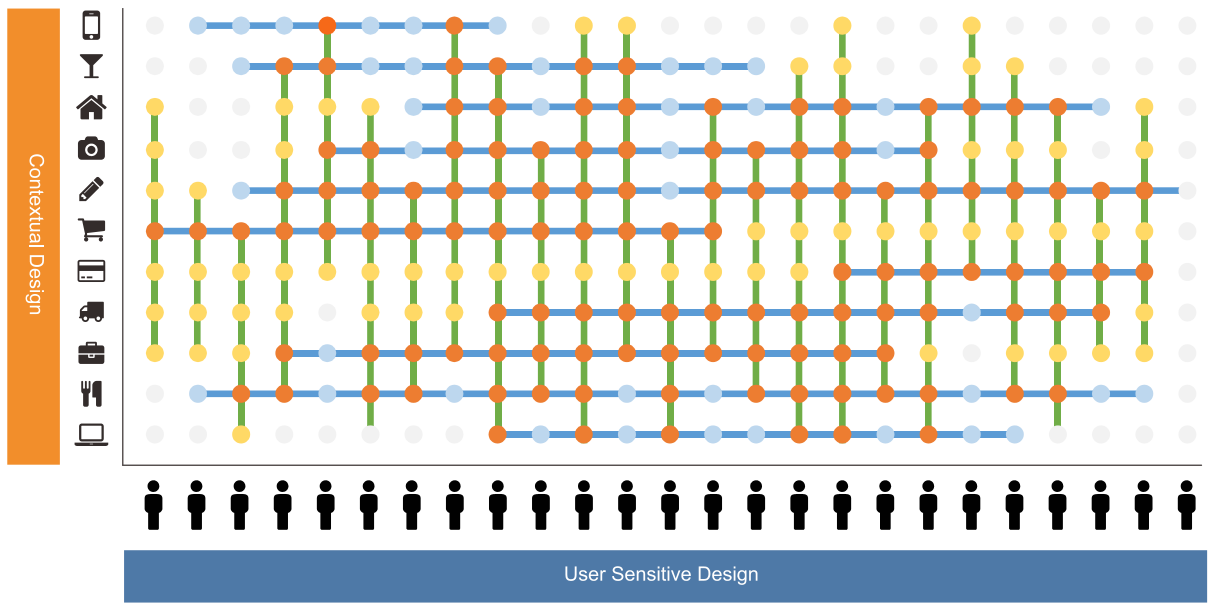

Fig. 3. Contextualized user-sensitive design map

In order to make the design meet the needs from different users in various contexts as much as possible, the authors argue that the priority goal in interaction design should not be to seek the best design for specific needs, instead, it should focus on how to provide individual users in different contexts with sufficient choices to accommodate their diverse needs under the established constraints, because the duplication and delivery cost of digital products is often as low as zero. Therefore, it is necessary to establish a contextualized user-sensitive design framework (Fig. 3). This framework should contain two core principles: one is to ensure that the individual user has a consistent experience in different contexts, and the other is to ensure that the differentiated needs and preferences of users who have different capabilities could be met equally.

The implementation of the framework is based on some preconditions and necessary system capabilities: for example, the system should be able to identify and record the characteristics of different users respectively, and be able to perceive different usage scenarios. Therefore, the perceptual design of the diversities of users and contexts will be incorporated into the whole design process, and we need to have in-depth understanding of these diversities. 


\section{User Diversities}

The emphasis on perceiving user diversities originates from the user-centered design research methodology. Since Jakob Nielsen put forward the concept of usability design[9] in the early 1990s, user research has been one of the central issues of interaction design. A concept similar to User-centered Design is Human-centered Design, which includes a wider range of stakeholders. The International Organization for Standardization formulated the Human-centered design processes for interactive systems (ISO 13407:1999)[10] in 1999. This standard was revised as Ergonomics of human-system interaction -- Part 210: Human-centered design for interactive systems ( ISO 9241-210:2010)[11], and has become the basis of many studies. However, as Ekaterina Novoseltseva pointed out in an article on UsabilityGeek.com, although it may be the good wishes of many designers and developers, in fact not all people will become actual users. Therefore, User-centered Design emphasizes more in-depth analysis of users than the Human-centered Design, focusing on the specific habits and preferences of the target users rather than just the general characteristics of people[12]. This is not to say that other stakeholders and environmental and sustainable factors are not important. No design methodology will cover all aspects of the design process. To study the specific habits and preferences of the target users, it is difficult to ignore the diversities of users. Here we focus on the diversities of users, which depend on our main purpose to reduce and eliminate the exclusion caused by diversities.

User diversities can be observed from two different perspectives. One is the individual differences within the user group. The other is the difference of the same user in different contexts. The latter can be regarded as a reflection of the context diversities projected on users, so we will discuss them together when we discuss context diversities in section 4 .

\subsection{Generalized User Balance Sheet}

Ergonomic user research usually starts with physiology, cognition and emotion, and divides physical factors into two aspects: motor and sensory[13]. The research on these factors often does not make a detailed distinction between different functional categories, but generally takes them as the basis for consideration in generating design proposals. However, from the perspective of the relationship between the behavior model and the aspects of human-computer system, different factors play completely different roles in human behavior. As stated in Fredrick Herzberg's hygiene-motivational factors theory[14], some are the motivational or incentive factors of behavior, and some are the hygiene factors of behavior. All designs for human-computer systems are ultimately to assist, support and promote users to complete purposeful behaviors, that is to say, the design activity itself is a purposeful behavior. If users are regarded as the core resources and partners of human-computer systems to complete behavioral activities, then different users will show different execution and cooperation willingness. It is the various user characteristics that determines the cooperation value and ability of users, and it is not static, but constantly changes with the target activities and contexts.

Many ergonomics researches are devoted to improving the execution ability of users in completing activities, which is based on the assumption that users have common understanding of the target activities envisioned by researchers and there is no lack of motivations: as long as users complete activities more easily, the efficiency of the system will be higher, and the user experience will be better. For the productivity equipment and tools with relatively simple usage scenarios, this assumption may not be a big problem. However, once the system is put into operation in a more complex real environment, changes in users and context make the hygiene factors and motivational factors work together on users' behaviors and are affected by more external factors. Not only does the users' behavior intention become at least as important as their behavior ability, but also the uncertainty of behavior ability is greatly increased. In view of this, we need to construct an effective analysis tool to accurately capture and evaluate user diversities. 
Since the user's behavior model conforms to the principles of economics and is inspired by finance, investment and accounting, the authors use financial statements as a reference, and introduces a "Generalized User Balance Sheet (GUBS)" to represent and reflect the user's attributes at a certain moment. We set a basic balance formula:

$$
\text { User Abilities (Assets) = User Requirements (Liabilities) + User Satisfaction (Equity) }
$$

Assets represent resources that can be used to accomplish a certain activity. These resources include not only the user's own physical function and cognition, but also emotional intelligence and available economic and social resources. We can simply understand them as the user's comprehensive ability. However, user liabilities include all factors that make users uncomfortable and need to be eliminated, such as unmet demand goals and unsatisfied preferences, all of which require extra efforts from users. User equity is the surplus of user's ability to cope with the demand. The more surplus this part has, the stronger the sense of pleasure and satisfaction, and the better the user experience.

According to a certain kind of classification, we can get a GUBS by properly arranging the specific items of users' abilities, needs/preferences and satisfaction at a certain moment.

The subjects and categories listed in Table 1 are just to show the general usage of this tool. Although there are many potential options, the actual user capabilities and needs involved in a product are usually limited. For specific practical projects, designers and researchers need to select and set appropriate subjects to accurately reflect the user-related capabilities, needs and preferences in their project.

Table 1. An example of Generalized User Balance Sheet

\begin{tabular}{ll}
\hline Assets & Liabilities \\
\hline Current Assets & Current Liabilities \\
Physical Function & Physical Needs \\
Motor & Economic Interest \\
Power & Profit \\
Agile & Risk \\
Sensory & Valuation \\
Visual & Non-Current Liabilities \\
Hearing & Cognitive Needs and Prefer- \\
& ences \\
Economic Capacity & Convenience \\
Income & Complexity \\
Purchasing Power & Emotional Preference \\
Investment Capacity & Taste \\
Risk Tolerance & Values/Beliefs \\
Credit & Character \\
Non-Current Assets & Habit \\
Cognitive Ability & Social Preference \\
Memory & Privacy \\
Language Ability & User Equity \\
Knowledge & Price Discount \\
Skills & Save Time \\
\hline
\end{tabular}

There are two main potential values in using the GUBS. First of all, design researchers can use it as a summary tool for user research results to describe the user's project-related capabilities and re- 
quirements, and use it as a basis for product design to determine the product's capability requirements and satisfaction degree for users. Secondly, the GUBS can also be used to identify and evaluate the diversities of different users in interactive product operation. Based on this, dynamic and parametric user profiles can be established. By comparing actual user data with design calibration values, and combining evaluation indexes and feedback of user experience, the reference standards for product optimization, iteration and dynamic response can be formed.

However, although the GUBS uses the form of accounting statements for reference, the types of user's preferences and abilities are very complex, and there is a lack of currency to convert different subjects in a unified way. Therefore, it cannot be used as an accurate quantitative tool at present. It is only helpful to grasp the user diversities on the whole and in individual classification, and to identify the individual diversities. However, the core significance lies in that, compared with other tools such as Persona, GUBS no longer describe users from a static perspective, but are based on dynamic models related to target tasks. Users' capabilities, needs and related equities are dynamic data that change at any time. Such user models are helpful to fit with a richer variety of usage situations and external environments, thus improving user experience and design inclusiveness.

\subsection{Multidimensional Analysis of Individual Diversities}

By using the GUBS or other various user research tools and methods, the individual diversities of target user groups can be analyzed and studied. In order to grasp the individual diversities of users more comprehensively, user research needs to explore users' capabilities and needs/preferences from multiple dimensions.

Physical. A large number of existing inclusive design studies focus on this dimension. The lost or weakened abilities of the main research objects - disabled and elderly groups - can mostly be attributed to physical functions. These functions are the original functional abilities of individuals. One type is motor functions, such as body type, (each part) muscle strength, muscle memory, speed, flexibility, agility, etc. It is the ability of individuals to feedback or take active actions to the external environment and other individuals through their own physical activities. The other is sensory ability, which is the individual's ability to obtain the external environment state and information. Usually, it is easy to naturally understand the sensory ability as vision, hearing, taste, touch, smell and so on possessed by the five senses, and at most it includes more detailed senses such as color, night vision, etc. These senses are processed by the brain to form perception. However, a broader sense of perception should include not only the acquisition of information on the state and changes of all external environments such as light, sound, temperature, taste, force, friction, etc. and the direct feelings of pain, itching, comfort, excitement, etc. by any individual not limited to human beings or even organisms, since they may become design objects or partners.

These capabilities are the biological basis of all behavioral capabilities, and are also the basic conditions for individuals to participate in competition and cooperation. Traditionally, the definition of disabled people in human society refers to the people who are lack of ability in these aspects. Low motor function will affect the user's ability to complete target activities. Low or even lack of sensory ability will reduce the input of external information and stimulation, which may affect the users' direct and indirect behavioral motivation. However, in some situations, the impact is not all negative. For example, people with visual impairment are less afraid of dark environment than other people. In this dimension, designers need to understand the relevant physical functions and sizes involved in the use of products, environment or services by target users, so as to make the design solution adapt to the size of as many people as possible, and try not to require users to have a high level of physical functions before normal use, or to provide assistive methods and tools to enhance users' functions in some aspects. Many research data and results of human factor engineering can be used for inclusive design in this dimension. Physical functions are independent and bound to 
individuals, which means that it is difficult for one individual to directly obtain and use functions possessed by other individuals. For severely disabled people and the disabling context, combining appropriate assistive technologies can effectively compensate for the loss of ability, and relying on other capabilities with compensatory function to complete the task is also a common design idea.

In addition to physical functions, physical dimensions also include many passive needs determined by non-subjective consciousness, such as instinct and conditioned reflex. Instinct is congenital demand and desire, highly related to human biological attributes, and is the most direct and basic source of demand. Reflex is an individual's non-subjective response to external environmental stimuli, including instinctive unconditioned reflex and acquired conditioned reflex. Unconditional reflex is the inherent fixed connection between external stimulus and individual response, while conditioned reflex is the temporary connection established between external stimuli and the individual response under certain conditions. It can be obtained and designed through training. The designer should pay attention to the habituation and degeneration of conditioned reflex. This kind of passive demand has relatively high regularity and predictability, and is suitable for empirical scientific research methods, and there are a large number of related disciplines (biology, physiology, psychology, ergonomics, etc.) research results and conclusions can be directly borrowed. The design for passive requirements is usually the bottom line and red line of inclusiveness, and its demand elasticity is very low. However, from the perspective of user diversities, the diversities in passive requirements are relatively limited, and different users have relatively high generality.

Cognitive. More and more products are becoming more and more complex while providing functions and experiences far beyond the past. From being able to know and use various functions provided by products to obtaining excellent user experience, users rely on mastering corresponding knowledge, information and skills. Respecting the users' existing cognition, experience and habits, and making the best use of the users' existing knowledge can make the products easier to understand and learned. Conversely, it will increase the users' burden, which will most likely lead to the users being forced to give up using the products. Sufficient information helps users to make choices that best meet their real needs. Incomplete information will lead to misjudgment and misoperation by users, resulting in adverse consequences. For example, insufficient information will lead to uncertainty expected by users. Uncertainty of behavior results is the risk cost for users. User will tend to increase the margin of safety, thus occupying too many resources.

The cognitive difference of users varies with age, experience and educational level, including both congenital cognitive ability difference and acquired experience, knowledge and skills difference. This makes the diversity in the ability and efficiency of different users in solving problems independently far greater than their physical differences, and even determines the success or failure of many activities. An experienced and skilled printing shop operator can easily accomplish several times the workload of a novice, while users lacking professional knowledge cannot even complete the basic operation of professional software. Distinguishing the difference between knowledge and skills is of great significance to ease the cognitive difference of users. Much knowledge is information that can be quickly known and used, while skills are the ability to complete a certain task that requires a certain amount of time to practice. In the process of interaction between products and users, necessary and lacking knowledge can be conveyed to users, thus eliminating obstacles of users. But for a particular skill, on the one hand, we should try our best to avoid the dependence of interactive operation on this skill, and at the same time, we should give users with this skill sufficient space to display it.

Emotional. Emotional dimension reflects the emotional value of products, environment and services and the user preferences. The emotional attribute of a product is an important feature that the product gets rid of "commodity attribute" and thus has non-materialized meaning. The core aspects 
of emotional dimension are aesthetic, cultural and psychological. The differences of users in these aspects are often diversified and changeable, which makes the inclusive design of this dimension full of uncertainty. Moreover, the biggest challenge to designers brought by user diversities in this dimension is that these differences often have obvious mutual exclusion, and the evaluation standard is not a single one. For example, some people's fondness is likely to be dismissed or even hated by others. Even if MUJI adopts neutral and simple design to avoid contradictions and conflicts, it cannot satisfy those consumers who are eager to make public and show off. In more complex multiuser platforms, users need to share more design elements, and emotional diversities between different users are more likely to produce conflicts and contradictions. In many cases, design also needs to respond to the consumer's demand for identity, and stimulate some demands by transmitting the value to satisfy consumer's needs, thus generating the desire to buy and use[15]. These rich and diverse needs are usually difficult to satisfy and are often contained by a single design. Fortunately, in most cases, the market has the same diversified supply capacity. Designers can make trade-offs for their own product positioning, so as to ensure that their design language is consistent with the target users, and can also provide different design for different users. The key is accurate matching between different designs and different users.

Economic. The economic dimension reflects the ability of users to exchange and control external resources. The economic ability of users not only restricts their access to products and services, but also influences their ability through leverage factors. Like a joker card, purchasing power can be easily converted into various other external capabilities by the market, thus greatly changing the actual situation of individuals. It is worth noting that the diversities in economic ability between different individuals is further enlarged compared with physical and cognitive diversities, and can also be doubled through credit leverage. However, economic ability does not necessarily affect consumers' value cognition. Good economic ability only gives consumers more freedom and leeway to make choice. In this sense, economic ability is a direct supporting factor for users' various needs. Diversities in economic ability will also bring about diversification of demand, thus generating more design and business opportunities.

Users' demands in the economic dimension are often at odds with users' capabilities in this dimension. Generally, users with poor economic capabilities are more sensitive to price and demand higher economic returns on products. Economic capability is not simply equivalent to an individual's financial situation. For a specific product, the user's willingness to bear risks and consumption ability on the product are the economic capability indicators related to the product design. Therefore, simple high-income groups and high-consumption "credit card slave" may have economic capability opposite to their comprehensive economic strength at a certain stage in a certain subdivision field.

\subsection{User-Sensitive Design}

Researchers from the Department of Applied Computing in University of Dundee took the lead in proposing the concept of user-sensitive and inclusive design[16, 17], the starting point of which is to bring the elderly and the disabled into the target user group of mainstream products. They think that some groups, such as the elderly, are different from ordinary users and designers, and have high diversity. The relevant user research often involves moral and ethical issues. It is difficult to copy the standard user-centered design methodology to sum up diverse user characteristics. There are not only substantial differences in user characteristics, but also changes in different time scales. User sensitive and inclusive design needs to respond to this dynamic diversity[18]. Compared with industry, the education field has always attached more importance to the implementation of the principle of teaching students according to their aptitude. With the concept of lifelong learning and a learning society being widely accepted, and information technology playing an increasingly important role in the learning process, individual learners are showing a more and more diversified 
trend, with different backgrounds, skills, interests, professional knowledge, goals and learning styles. In order to meet such diverse requirements, user-centered design and user-sensitive design have become logical choices[19].

Providing an intelligent user interface is an effective means to make products flexible to personalized needs. The framework to deal with user heterogeneity in the early stage envisages two ways: one is the Adaptable System, i.e. the system allows users to customize. The second is the Adaptive System, that is, the system can adjust the appearance and behavior of the interface according to the characteristics of the user[20]. Although this framework was proposed long before the popularity of graphical user interfaces, it is worthy of respect that the idea of starting from both passive and active aspects still has important guiding significance. We can see that the same thinking is still practiced in the latest interactive design of mobile devices and applications.

How to cooperate with specific users in the best way is the core concern of user sensitivity design. It is an ideal scheme to achieve this goal, which has both adaptability and adaptability. To do this, we first need to be able to identify different users. No matter whether login mechanism or anonymous method is adopted, only by separating unique individuals from a wide range of user groups can targeted responses be made to users. After identifying the user, the system needs to judge the degree of understanding of the user, which depends on the system's archiving of the user's profile. If the user's archived complete information is available, the system can match the ideal preset value for the user through adaptive design. On the contrary, if the system identifies a new unfamiliar user, the system can gradually establish the user's profile through novice guidance or other adaptive design methods in the process of interaction with the user (Fig. 4). From this we can conclude that a user-sensitive design system must include the following features:

- User identification capability;

- Archiving system for user profiles and data;

- Adaptive design of system interface and function;

- The system interface and functions can be customized.

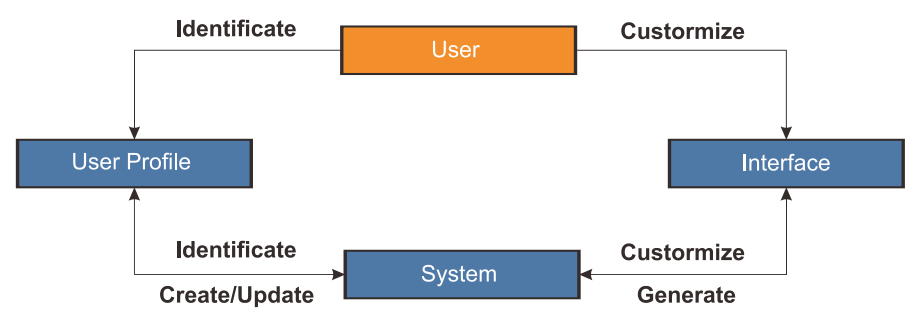

Fig. 4. User-Sensitive Design Framework

\section{Contextual Diversities}

In addition to individual diversities among users, the diversities that users show due to changes in constraints in different contexts may exceed all others. Even though attention has been paid to the diversities between individual users, if the influence of interaction between users and external environment in different contexts is ignored, contextual exclusion will occur.

\subsection{A Cases Study of Contextual Diversities in User Research}

Method. In order to analyze and understand the relationship between demand and constraints in user research, the author conducted a survey on MRT (Mass Rapid Transit) payment methods. The survey distributed questionnaires through mobile social networks, and received 519 visits and 201 valid answers. Affected by the media, 200 of them were filled in through mobile phones and only 1 
was completed on computers. The purpose of our research is not to understand the user needs reflected in the questionnaire itself and its representation to the real user population, but to study the differences between the individual user needs in the actual environment and the ideal environment, and how to understand the differences and translate them into design insights. Therefore, the data collected by this questionnaire is sufficient for our research.

Result. The first question in the questionnaire is about how often users travel by MRT. The users who choose to travel daily and several times a week account for $22.89 \%$ and $31.84 \%$ respectively, which together account for more than half of the total. They represent the user group that regards MRT as one of the main modes of travel. The proportion of respondents who choose to take several times a month and occasionally is $16.42 \%$ and $26.37 \%$ respectively. They can be regarded as lowfrequent users with experience of MRT. Only $2.49 \%$ of the respondents said that they basically did not take MRT. We will mainly examine their demand characteristics in the ideal environment. Considering that more than half of the respondents come from Shanghai and Beijing, where MRT is well developed (judging by IP addresses, accounting for $47 \%$ and $5 \%$ of the respondents respectively), this result basically meets our expectations.

The questionnaire asked other users except those who basically do not take MRT what kind of fare collection and payment methods they mainly use when taking MRT. The available answers include "Pay-as-you-go Card", "One-way Ticket", "QR Code", "NFC", "Wearable Device" and a "Other" option that can be filled in by users. The results show that only one interviewee has chosen the "Other" option and filled it in as "AliPay". The answer can actually be classified as "QR Code", which proves that the options provided by the questionnaire basically cover all ticket checking and payment methods currently provided by China's MRT. Specifically, 122 users chose "Pay-as-yougo Card", accounting for $62.2 \%$ of the respondents, far ahead of other options and in a dominant position. QR Code was the second choice, with 82 places, accounting for $41.8 \%$. Some mobile phone models with NFC support contactless payment, with 44 users using this relatively "advanced" method accounting for $22.4 \%$; Only $13.3 \%$ of the respondents used the most basic and "primitive" one-way ticket. Emerging wearable devices such as bracelets and watches are probably still in the market introduction stage, with the overall user share being the lowest, at $4.1 \%$.

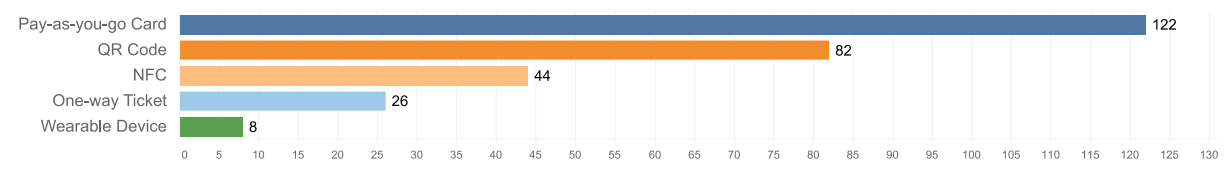

Fig. 5. Number of passengers of various payment methods.

It is worth noting that the answer to this question is actually not the choice made by the interviewee when answering the question, but the expression of their actual choices under real constraints in real life. These actual choices are the optimal solution that the user chooses under the rational guidance after considering all real costs, convenience and potential benefits in a long period of time and putting it into action. Although the reality constraint is not easy for us to perceive and attach importance to because it is pervasive, its influence undoubtedly exists. Users' choices tend to converge at the equilibrium point of supply and demand. In this multi-choice question, the total proportion of all the respondents' choices is $143.9 \%$, which reflects that some respondents - in fact, $31.6 \%$ of them will use more than one method of payment. This may be due to the fact that the same people will encounter different usage contexts and have different preferences for different contexts, but what we see is that more people $(68.4 \%)$ choose to use the only fare collection and payment method that is, the best method they choose under the constraints of reality. 


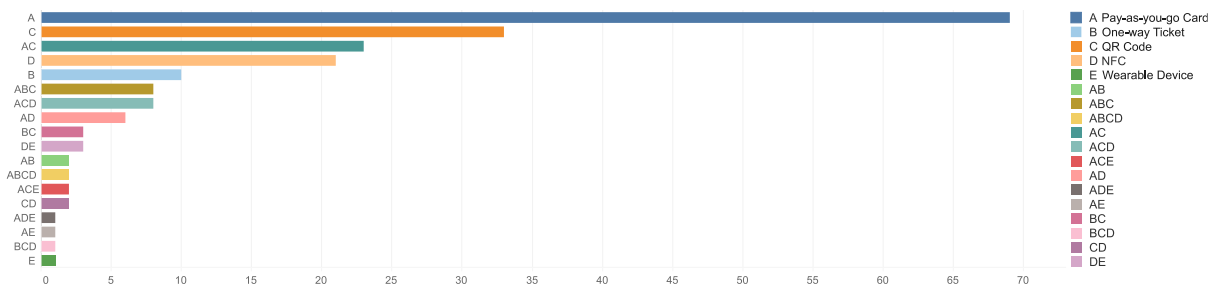

Fig. 6. Number of passengers adopted by various payment combinations.

The questionnaire then asked them to choose their ideal payment method. In addition to the original options, we have provided "Identity Card", "Fingerprint Identification", "Face Recognition" and "Voice Recognition" as new options for the four existing payment methods that have not yet been adopted but for which related technology implementation solutions already exist. This question provided more options. However, since this topic is multiple choice, we believe that the new options will not in theory unduly dilute the respondents' positive evaluation of the existing payment methods.

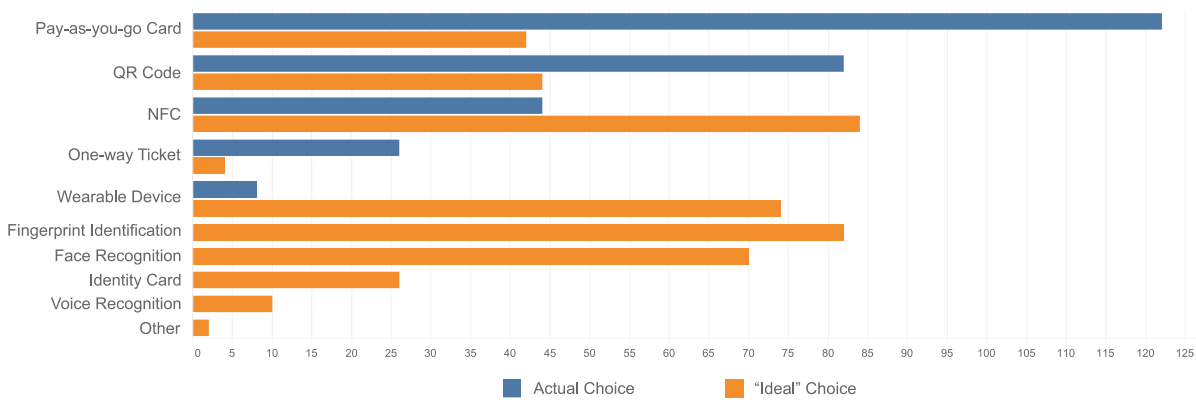

Fig. 7. Comparison of actual payment methods and "ideal" payment methods

Findings. We found an interesting result when we compared the daily payment method previously selected by the user with the "ideal" payment method. We have not made any improvement to the existing payment method, but only asked the respondents to make a new choice by asking questions instead of retelling the actual behavior. However, the results showed that only $20.9 \%$ of the respondents thought that the pay-as-you-go card used by the most respondents $(62.2 \%)$ in daily life was the ideal way, and the number of people who choose QR code and one-way Tickets has dropped significantly. On the contrary, in reality, only $22.4 \%$ and $4.1 \%$ of the respondents use NFC and wearable devices respectively, $41.8 \%$ and $36.8 \%$ of the respondents think that they are ideal methods, which have become the two most popular choices among the existing payment methods. Among the four new options, fingerprint recognition and face recognition have achieved relatively high acceptance, which is roughly equivalent to contactless payment of mobile phones and wearable devices, while ID card recognition and voice recognition have not been recognized by most respondents.

The main difference between the two choices is that in the new choice, what we have set is a virtual context. Users do not need to bear any actual costs for their choices, but only need to express their ideal demands. This is similar to the context that researchers will unconsciously construct in most user researches based on questionnaires and interviews. We can see how different users' choices are from the real behaviors in the real context under this virtual context. On the one hand, it shows that the existing research results of users based on virtual contexts need to be evaluated more prudently. On the other hand, it also shows that users' needs and preferences are by no means a stable and static attribute. Any prediction and assumption about users' behaviors and choices depends on clear contextual constraints. 


\subsection{Contextual Design}

Contextual design has been studied and applied in computer hardware[21] and software[22] systems for a long time. In mainstream object-oriented programming languages, functions are triggered by contextual events, which has become a common basic knowledge. However, in the traditional industrial and architectural fields, most products and spaces are not designed for multi-context and complex environment, or the early design research paid little attention to this kind of design. When entering the 21 st century, more and more information technologies are integrated into product design, the boundaries between hardware and software systems begin to blur gradually, and multifunctional and multi-mode designs suitable for different contexts gradually increase, thus contextual design has begun to receive more and more attention.

Holtzblatt and Beyer summarized a series of key principles and design processes of contextual design[8], but that process may not substantially differ from other versions of interactive design processes. The focus of their attention is how to understand the user's demands and characteristics from the usage context, so that the design can help users to accomplish the task better. What we want to argue is that the key problem in contextual design is how the product responds to complex and uncertain context changes in real time and accurately. Therefore, through field investigation, user participation and other methods to clarify the needs of users at the design stage can certainly provide valuable insights for design research output, but it is not sufficient to provide sufficient design basis for contextual design. If the product has enough ability to help users accomplish tasks, but cannot coordinate all functions and resources in specific contexts and enter the best mode for specific tasks, then the original intention of contextual design, that is, to ensure users have consistent experience under diverse context, has still not been realized.

Contextual design does not need to reorganize and rebuild a unique design process. Similar to user-sensitivity design, what we need to do is to establish corresponding models for different contexts and usage scenarios, and design corresponding tools and interfaces for system perception of context changes. When the system senses the change of the user's usage context, it needs to be able to respond to the change and adjust the system to adapt to the change of the context. This adjustment mechanism is the focus of contextual design. The specific adjustment mechanism varies according to the relevant subdivisions of the actual project. It is difficult for us to carry out the detailed adjustment mechanism here because of the limited space. However, the authors believe that this topic has a broad space for research and discussion. In addition, even if we have designed an almost perfect adjustment mechanism, we cannot guarantee that it can fully meet the needs of users in that context, so it is always necessary and valuable to give users convenient intervention and adjustment ability to the system.

\section{Contextualized User-Sensitive Design}

After discussing user-sensitive design and contextual design respectively, it is possible to combine the two and summarize a design framework that can be applied to diverse users in diverse contexts. Through user research in early stage, we can initially understand the distribution of user diversities and context diversities, and establish a preliminary user balance sheet, story board and scenario model for the target users. By cross-examining the diversities between users and contexts, designers and researchers can find the gaps and blind areas of product's inclusion to users in different usage contexts (Fig. 3). These gaps and blind areas can be used as benchmarks for further design improvement. 


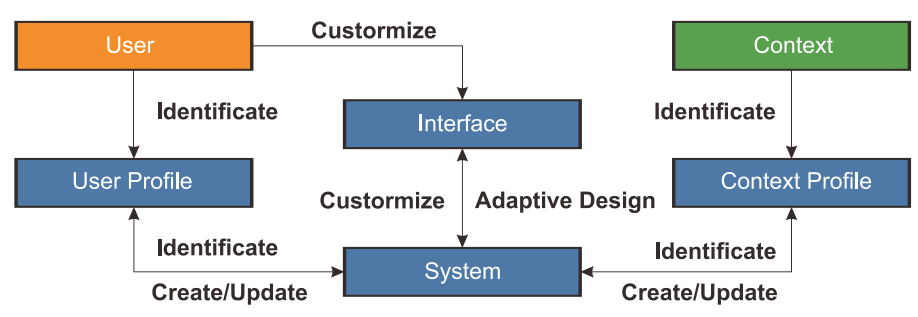

Fig. 8. Contextualized User-Sensitive Design Framework

As mentioned earlier, the implementation of the framework is based on some prerequisites and necessary system capabilities: for example, the system should be able to identify and remember the characteristics of different users and perceive different contexts and scenarios. Consequently, the design of identifying user and context diversities will be integrated into the whole design process. On this basis, the modeling of users and scenarios will go on from design and development till daily operation and maintenance after launch when users are continuously using the product. The target user's persona and scenario storyboards are established, and then the profiles of users and scenarios are created and accurately refined with the data generated by the user's interaction with the system. Thanks to the rapid growth of data calculation and storage capacity, the user profiles and scenario descriptions can be subdivided into a greater extent than ever before. It is even possible to maintain a dynamically updated accurate profile for each user and scenario.

One of the major limitations of the previous product and interaction design is that the granularity of the design is very large, and there are often only a limited number of design schemes to deal with different context, which greatly limits the inclusion of the design to user diversities and context changes. In the framework of contextualized user-sensitive design, in order to match accurate user and context perception ability, the final output of contextualized user-sensitive interaction design will no longer be implemented into a standardized GUI, but rather a parameterized and algorithmic interface and interaction experience. Responsive design is only a partial and preliminary attempt of this kind of design. Through the matching mechanism and algorithm designed in advance by designers, combined with user customization and the application of artificial intelligence technology with broad prospects, the design granularity of digital products will be greatly reduced, thus seamlessly connecting the changing user needs and capabilities. Users will not only obtain completely differentiated and customized content and services, but also include completely differentiated and customized interface and interactions, which keep consistent across different contexts.

\section{Conclusion}

This paper constructs a contextualized user-sensitive design framework by analyzing the interaction between behavior models and various aspects in human-computer systems. The design framework is based on two basic dimensions of users and contexts, and studies the diversities and changing factors in human-computer systems. In order to reflect the multi-dimensional dynamic characteristics of users, the authors propose a new user research and analysis tool, the generalized user balance sheet, and use a questionnaire case to reveal the huge differences of users' needs in different contexts. Based on the contextualized user-sensitive design framework, this paper shows the basic methods and essential system elements of user-sensitive design and contextual design, as well as the preliminary exploration of combining the two for inclusive design.

The authors believe that when the interaction design of digital products can respond to the differentiated needs and preferences of each user contextually, it is close to achieving the maximum inclusion. Its significance lies in making full use of the possibilities created by technological progress, so that each individual will no longer compromise with the need to share the same interface and design resources with others, thus expanding inclusion. 


\section{References}

1. Gonzalez, I. and P. Morer, Ergonomics for the inclusion of older workers in the knowledge workforce and a guidance tool for designers. Applied Ergonomics, 2016. 53, Part A: p. 131-142 (2016).

2. Fogg, B., A behavior model for persuasive design, in Proceedings of the 4th International Conference on Persuasive Technology. ACM: Claremont, California, USA. p. 1-7 (2009).

3. Mises, L.v., Human Action: A Treatise on Economics. Shanghai: Shanghai Academy of Social Sciences Press (2015).

4. Miaskiewicz, T. and K.A. Kozar, Personas and user-centered design: How can personas benefit product design processes? Design Studies, 2011. 32(5): p. 417-430 (2011).

5. Pfeil, U., R. Arjan, and P. Zaphiris, Age differences in online social networking - A study of user profiles and the social capital divide among teenagers and older users in MySpace. Computers in Human Behavior, 2009. 25(3): p. 643-654 (2009).

6. Marcotte, E. Responsive Web Design. (2010).

7. Gustafson, A., Adaptive Web Design: Crafting Rich Experiences with Progressive Enhancement. 2nd ed. USA: New Riders (2016).

8. Beyer, H. and K. Hultzblatt, Contextual Design: Defining Customer-Centered Systems. San Francisco: Morgan Kaufmann (1998).

9. Nielsen, J., Usability Engineering. 1st ed.: Morgan Kaufmann (1993).

10. ISO 13407:1999, in Human-centred design processes for interactive systems. International Organization for Standardization (1999).

11. ISO 9241-210:2010, in Ergonomics of human-system interaction -- Part 210: Human-centred design for interactive systems. International Organization for Standardization (2010).

12. Novoseltseva, E. User-Centered Design: An Introduction. https://usabilitygeek.com/user-centereddesign-introduction/, last accessed 2019/1/5

13. Gonzalez, I. and P. Morer, Developing a workstation design assistance tool for older knowledge workforce inclusion. Universal Access in the Information Society, 2017. 16(3): p. 641-651 (2017).

14. Herzberg, F., B. Mausner, and B.B. Snyderman, The Motivation to Work. New Jersey, USA: John Wiley \& Sons (1959).

15. Sparke, P., An introduction to Design and Culture. Nanjing, China: Yilin Press, Ltd (2012).

16. Newell, A.F. and P. Gregor, "User sensitive inclusive design" - in search of a new paradigm, in Proceedings on the 2000 conference on Universal Usability. ACM: Arlington, Virginia, USA. p. 3944 (2000).

17. Newell, A.F., et al., User-Sensitive Inclusive Design. Universal Access in the Information Society, 2011. 10(3): p. 235-243 (2011).

18. Gregor, P., A.F. Newell, and M. Zajicek, Designing for dynamic diversity: interfaces for older people, in Proceedings of the fifth international ACM conference on Assistive technologies. ACM: Edinburgh, Scotland. p. 151-156 (2002).

19. Glavinic, V. and A. Granic, HCI Research for E-Learning: Adaptability and Adaptivity to Support Better User Interaction, in Hci and Usability for Education and Work, Proceedings, A. Holzinger, Editor. p. 359-+ (2008).

20. Adaptive User Interfaces: Principles and Practice, ed. S.-H. Matthias, M. Uwe, and K. Thomas. New York, NY, USA: Elsevier Science Inc. 362 (1993).

21. Curtis, P., et al., Customer-focused design data in a large, multi-site organization, in the SIGCHI Conference on Human Factors in Computing Systems: the CHI Is the Limit. ACM Press: Pittsburgh, Pennsylvania, United States. p. 608-615 (1999).

22. Rockwell, C., Customer connection creates a winning product: building success with contextual techniques. Interactions, 1999. 6(1): p. $50-57$ (1999). 